\title{
Effect of novel dithiooctanoate monomers, in comparison with various sulfur- containing adhesive monomers, on adhesion to precious metals and alloys
}

\author{
Kunio IKEMURA 1 , Katsunori KOJIMA², Takeshi ENDO ${ }^{3}$ and Yoshinori KADOMA ${ }^{2}$ \\ ${ }^{1}$ Department of Research and Development, Shofu Inc., 11 Kamitakamatsu-cho, Fukuine, Higashiyama-ku, Kyoto 605-0983, Japan \\ ${ }^{2}$ Department of Applied Functional Molecules, Division of Biofunctional Molecules, Institute of Biomaterials and Bioengineering, Tokyo Medical and \\ Dental University, 2-3-10 Kanda-Surugadai, Chiyoda-ku, Tokyo 101-0062, Japan \\ ${ }^{3}$ Molecular Engineering Institute, Kinki University, 11-6 Kayanomori, lizuka, Fukuoka 820-8555, Japan \\ Corresponding author, Kunio IKEMURA; E-mail: k-ikemura@shofu.co.jp
}

\begin{abstract}
This study investigated the effect of novel dithiooctanoate monomers, in comparison with conventional sulfur-containing monomers, on adhesion to precious metals and alloys. Nine experimental primers containing $5.0 \mathrm{wt} \% 2$-methacryloyloxyethyl 6,8-dithiooctanoate (2-MEDT), 6-methacryloyloxyhexyl 6,8-dithiooctanoate (6-MHDT), 10-methacryloyloxydecyl 6,8-dithiooctanoate (10-MDDT), bis(2methacryloyloxyethyl) disulfide (BMEDS), bis(5-methacryloyloxypentyl) disulfide (BMPDS), bis(10-methacryloyloxydecyl) disulfide (BMDDS), 6-(4-vinylbenzyl- $n$-propyl) amino-1,3,5-triazine-2,4-dithione (VBATDT), $N$-(4-mercaptophenyl)methacrylamide (MPMA), or 4-methacryloyloxyethoxycarbonylphthalic anhydride (4-META; control) were prepared. After primer pretreatment and bonding using modified MMA-PMMA/BPO-DEPT resin, tensile bond strengths to precious metals and alloys after 2,000 thermal cycles were measured. For bonding to Au or Ag, novel 2-MEDT, 6-MHDT, and 10-MDDT exhibited significantly higher tensile bond strengths than conventional BMEDS, BMPDS, BMDDS, VBATDT, MPMA, and 4-META $(p<0.05)$. For bonding to Au alloy, Ag alloy, and $\mathrm{Au}-\mathrm{Ag}-\mathrm{Pd}$ alloy, all the novel dithiooctanoate monomers showed significantly higher tensile bond strengths than conventional BMEDS, BMPDS, VBATDT, MPMA, and 4-META $(p<0.05)$. It was found that novel dithiooctanoate monomers exhibited excellent bonding to precious metals and alloys when compared with conventional sulfur-containing monomers.
\end{abstract}

Keywords: Dithiooctanoate monomer, Sulfur-containing adhesive monomer, Precious metal, Dental precious metal alloy

\section{INTRODUCTION}

In prosthetic dentistry, resin-veneered prostheses are now frequently used in dental practice ${ }^{1}$. The use of metal primer in the fabrication of resin composite-veneered restorations and intraoral repair of fractured ceramic or resin composite veneers has increased substantially, mainly because of the development of adhesionpromoting monomers (adhesive monomers) for dental casting alloys and ceramics ${ }^{2}$. Bonding to non-precious metals and alloys has been progressed considerably ${ }^{3)}$, mainly by the use of metal primers which contain acidic adhesive monomers ${ }^{4-8)}$ and the employment of surface modification techniques ${ }^{9 \cdot 11}$. In contrast, bonding of acidic adhesive monomers to precious metals and alloys has been characterized by low bond strengths due to the poor water resistance of the adhesive systems ${ }^{12)}$ or the chemical inertness of precious metals and alloys ${ }^{13}$. To achieve strong adhesion, numerous surface modification methods have been developed —such as heat treatment ${ }^{12,14)}$, electroplating with $\operatorname{tin}^{15,16)}$, silica coating ${ }^{9,11,17)}$, ion-coating ${ }^{12)}$, and the application of liquid Ga-Sn alloy ${ }^{18)}$.

Over the past decade, relentless efforts to improve the bonding technology of dental adhesives to precious metals and alloys have yielded major advances, notably the development of sulfur-containing adhesive monomers which contain at least one sulfur atom in their structures. Sulfur-containing adhesive monomers boast of two key advantages that are highly desirable: simple application with effective adhesion. These advantages stem from their ability to form chemical bonds with metal atoms on the surfaces of precious metals ${ }^{19,20)}$, due to the chemical adsorption of organic sulfur compounds on gold surfaces and the formation of monolayer films by a spontaneous assembly of organic thiols ${ }^{21,22)}$. Thereby, numerous sulfur-containing adhesive monomers ${ }^{13,23-25)}$ were developed - such as thiophenols, thiophosphoric acids, triazinedithiones, thiols, thiobarbituric acids, sulfides, disulfides, and thiouracil and thiirane monomers. Some of these sulfur-containing monomers were already included as an extremely valuable component in the formulations of some commercial metal primers for bonding to dental precious metal alloys. Incidentally, most of sulfur-containing adhesive monomers were developed in Japan, and that most of the studies which pertained to bonding to precious metals and alloys using sulfur-containing monomers were conducted by Japanese researchers. The research and development of sulfurcontaining adhesive monomers is still continuing unabated in Japan.

In our quest for more advanced sulfur-containing adhesive monomers, eight kinds of novel acryloyloxyalkyl and methacryloyloxyalkyl 6,8-dithiooctanoates (dithiooctanoate monomers) were previously synthesized ${ }^{26)}$. It was found that novel dithiooctanoate monomers exhibited excellent bonding performances to precious metals and alloys using methyl methacrylatepolymethyl methacrylate/tri- $n$-butylborane oxide (MMA-PMMA/TBBO) as the adhesive resin ${ }^{26)}$. In light of this encouraging result, our research strategy further focused an attractive target which was to compare the 
bonding abilities of dithiooctanoate monomers ${ }^{26)}$ to precious metals and alloys against those of conventional sulfur-containing monomers, such as thiophenols ${ }^{23)}$, disulfides ${ }^{24)}$, and triazinedithiones ${ }^{25)}$.

Therefore, the aim of the present study was to investigate the effect of three kinds of novel dithiooctanoate monomers on adhesion to precious metals and alloys [namely, gold (Au), silver (Ag), platinum (Pt), palladium (Pd), Au alloy, Ag alloy, and Au-Ag-Pd alloy] using a modified MMA-PMMA resin having benzoyl peroxide (BPO)- $N, N$-di(2-hydroxyethyl)$p$-toluidine (DEPT) as a polymerization initiator system. The obtained bond results were then compared with five kinds of conventional sulfur-containing adhesive monomers (namely, three disulfides, one triazinedithione, and one thiophenol) and one acidic monomer.

\section{MATERIALS AND METHODS}

Preparation of adhesive monomers

Figure 1 shows the chemical structures of sulfurcontaining adhesive monomers (Figs. 1a-d) and 4-META (Fig. 1e) used in this study. For the dithiooctanoate monomers (Fig. 1a) -namely, 2-methacryloyloxyethyl 6,8-dithiooctanoate (2-MEDT), 6-methacryloyloxyhexyl 6,8-dithiooctanoate (6-MHDT), and 10methacryloyloxydecyl 6,8-dithiooctanoate (10-MDDT), they were synthesized by an esterification reaction between 6,8-dithiooctanoic acid and 2-hydroxyethyl methacrylate, 2-hydroxyhexyl methacrylate, or 10-hydroxydecyl methacrylate respectively according to the method reported in our previous study ${ }^{26)}$. For the non-cyclic disulfide monomers (Fig. 1b) -namely, bis(2methacryloyloxyethyl) disulfide (BMEDS), bis(5methacryloyloxypentyl) disulfide (BMPDS), and bis(10- (a) Dithiooctanoate monomers<smiles>C=C(C)C(=O)OC(C)(C)CC(C)(C)OC(=O)C(C)(C)CC1(C)CCSS1</smiles>

$\mathrm{n}=2:$ 2-MEDT

$\mathrm{n}=6:$ 6-MHDT $\mathrm{n}=10:$ 10-MDDT (b) Disulfide monomers<smiles>C=C(C)C(=O)OC(C)(C)CC(C)(C)SSC(C)(C)CC(C)(C)OC(=O)C(=C)C</smiles>

$\mathrm{n}=2$ : BMEDS $\mathrm{n}=5$ : BMPDS $\mathrm{n}=10:$ BMDDS

(c) Triazinedithione: VBATDT<smiles></smiles>

Thione form

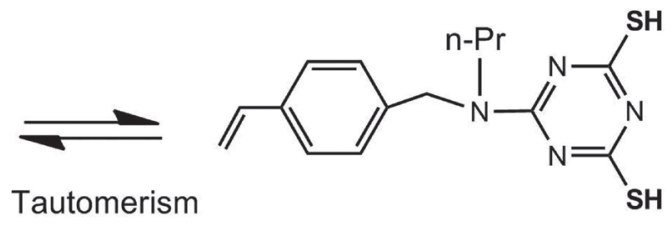

Thiol form (d) Thiophenol<smiles>C=C(C)C(=O)Nc1ccc(S)cc1</smiles>

MPMA (e) Non sulfur-containing monomer<smiles>C=C(C)C(=O)OCCOC(=O)c1ccc2c(c1)C(=O)OC2=O</smiles>

4-META

Fig. 1 Schematic illustration of the chemical structures of sulfur-containing adhesive monomers and non sulfurcontaining monomer used in this study. 2-MEDT: 2-methacryloyloxyethyl 6,8-dithiooctanoate, 6-MHDT: 6-methacryloyloxyhexyl 6,8-dithiooctanoate, 10-MDDT: 10-methacryloyloxydecyl 6,8-dithiooctanoate, BMEDS: Bis(2-methacryloyloxyethyl) disulfide, BMPDS: Bis(5-methacryloyloxypentyl) disulfide, BMDDS: Bis(10methacryloyloxydecyl) disulfide, VBATDT: 6-(4-vinylbenzyl- $n$-propyl)amino-1,3,5-triazine-2,4-dithione, MPMA: $\mathrm{N}$-(4-mercaptophenyl)methacrylamide, 4-META: 4-methacryloyloxyethoxycarbonylphthalic anhydride. 
methacryloyloxydecyl) disulfide (BMDDS), they were prepared as previously reported ${ }^{24)}$. Similarly, for triazinedithione [6-(4-vinylbenzyl- $n$-propyl)amino-1,3,5triazine-2,4-dithione (VBATDT); Fig. 1c] ${ }^{25)}$, thiophenol [ $N$-(4-mercaptophenyl)methacrylamide (MPMA); Fig. $1 \mathrm{~d}]^{23)}$, and non sulfur-containing monomer [4-methacryloyloxyethoxycarbonylphthalic anhydride (4-META); Fig. 1e] ${ }^{27)}$, they were prepared as previously reported.

\section{Preparation of other reagents}

Methyl methacrylate (MMA), polymethyl methacrylate (PMMA), and 2-hydroxyethyl methacrylate (HEMA) were purchased from Mitsubishi Rayon Co. Ltd. (Tokyo, Japan). Poly(ethyl methacrylate) (PEMA) (MW=80,000) and PMMA-PEMA copolymer were purchased from Negami Chemical Industrial Co. (Tokyo, Japan). Ethylene glycol dimethacrylate (EGDMA) was purchased from Shin-Nakamura Chemical Co. Ltd. (Wakayama, Japan). Benzoyl peroxide (BPO) was purchased from Kawaguchi Chemical Co. Ltd. (Tokyo, Japan). Butylated hydroxytoluene (BHT) and N,N-di(2-hydroxyethyl)-ptoluidine (DEPT) were purchased from Wako Pure Chemical Co. Ltd. (Tokyo, Japan) and used without further purification. Silane-treated silica fillers (mean particle size: $1.5 \mu \mathrm{m}$ ) were prepared using $\gamma$-methacryloyloxypropyltrimethoxysilane (KBM-503; Shin-Etsu Chemical Co. Ltd., Tokyo, Japan).

Preparation of experimental adhesive primers and adhesive resin

Nine kinds of experimental adhesive primers, whereby each primer contained $5.0 \mathrm{wt} \%$ of a dithiooctanoate monomer (i.e., 2-MEDT, 6-MHDT, or 10-MDDT), a non-cyclic disulfide monomer (i.e., BMEDS, BMPDS, or BMDDS), or a sulfur-containing monomer (i.e., VBATDT or MPMA) in acetone, were prepared. For comparison purpose, a control primer containing $5.0 \mathrm{wt} \%$ of 4 -META as a non sulfur-containing monomer was prepared.

For the experimental adhesive resin, it was prepared based on the composition listed in Table 1 . The powder was prepared using PMMA, PEMA, PMMA-PEMA copolymer, silane-treated silica fillers, and BPO. The liquid was prepared using MMA, HEMA, EGDMA, DEPT, and BHT. Since silane-treated silica fillers, EGDMA, and HEMA were added to MMA-PMMA resin, the experimental adhesive resin was hence termed as

\section{“modified” MMA-PMMA/BPO-DEPT resin.}

\section{Preparation of metal adherends}

Four kinds of precious metals [namely, gold (Au), silver $(\mathrm{Ag})$, platinum $(\mathrm{Pt})$, and palladium $(\mathrm{Pd})]$ used as precious metal adherends were purchased from ISHIFUKU Metal Industry Co., Ltd. (Tokyo, Japan). The dental precious metal alloys used in this study were namely, Au alloy (Casting Gold M.C. Type IV, GC Dental Products Corp., Tokyo Japan), Ag alloy (Sunsilver, Sankin Kogyo Co. Ltd., Tokyo, Japan), and Au-Ag-Pd alloy (Castwell M.C., GC Dental Products Corp., Tokyo, Japan). Their compositions were as per those given in a previous report ${ }^{18)}$.

\section{Tensile bond strength test}

The surface of each metal adherend was abrasively finished with alumina sandpapers of 1,000, 2,000, and 4,000 grits. An experimental primer was coated on each metal surface. Each primer-coated metal adherend was ultrasonically cleaned in acetone and allowed to stand overnight in acetone. After which, it was further cleaned ultrasonically in acetone and dried. On each metal adherend surface, the adhesion area was defined using a cellophane tape with a 5 -mm perforation. The powder and liquid of modified MMA-PMMA/BPO-DEPT resin were mixed together at a powder/liquid ratio of 2.5/1, and then the resin was chemically cured for 5.5 minutes at $23 \pm 1^{\circ} \mathrm{C}$ on the adhesion area of each primer-treated metal adherend surface. For bonding durability evaluation, the bonded specimens were subjected to a thermal cycling test of 2,000 cycles between water baths of $4^{\circ} \mathrm{C}$ and $60^{\circ} \mathrm{C}$ in alternate one-minute intervals. Tensile bond strength measurements were made on five specimens for each metal with a universal testing machine (Autograph AGS-1000A, Shimadzu Co., Kyoto, Japan) at a crosshead speed of $2.0 \mathrm{~mm} / \mathrm{min}$ and at $23 \pm 1^{\circ} \mathrm{C}$.

\section{Statistical analysis}

Fractured surfaces after tensile bond strength testing were not examined in this study. Mean and standard deviation for the load at failure were calculated and the results subjected to one-way analysis of variance (ANOVA), followed by Newman-Keuls multiple comparison test at $5 \%$ confidence level.

Table 1 Compositions of the powder and liquid of experimental modified MMA-PMMA/BPO-DEPT resin used in this study

\begin{tabular}{lclr}
\hline Powder $^{\phi}$ & $(\mathrm{wt} \%)$ & \multicolumn{1}{c}{ Liquid $^{\phi}$} & $\left(\mathrm{wt}_{\mathrm{O}} \mathrm{)}\right)$ \\
\hline polymethyl methacrylate [PMMA] & 35.0 & methyl methacrylate [MMA] & 91.5 \\
poly(ethyl methacrylate) [PEMA] & 13.0 & 2-hydroxyethyl methacrylate & 5.0 \\
PMMA-PEMA copolymer & 39.0 & ethylene glycol dimethacrylate & 2.0 \\
Si-treated silica powder & 12.3 & N,N-di(2-hydroxyethyl)- $p$-toluidine [DEPT] & 1.2 \\
benzoyl peroxide [BPO] & 0.7 & butylated hydroxytoluene & 0.3 \\
\hline
\end{tabular}

${ }^{\phi}$ Powder/Liquid ratio: 2.5/1. 


\section{RESULTS}

Tables 2 and 3 respectively present the tensile bond strengths of modified MMA-PMMA/BPO-DEPT resin to precious metals (i.e., $\mathrm{Au}, \mathrm{Ag}, \mathrm{Pt}$, and $\mathrm{Pd}$ ) and precious metal alloys (i.e., Au alloy, Ag alloy, and Au-Ag-Pd alloy), using nine experimental primers respectively containing 2-MEDT, 6-MHDT, 10-MDDT, BMEDS, BMPDS, BMDDS, VBATDT, MPMA, or 4-META (control) after 2,000 thermal cycles. It could be seen in Tables 2 and 3 that the control acidic adhesive monomer, 4-META, exhibited either $0 \mathrm{MPa}$ bond strength or poor adhesion to both precious metals and alloys. In sharp contrast, all the three novel dithiooctanoate monomers exhibited significantly higher bond strengths than 4-META $(p<0.05)$.

On bonding to $\mathrm{Au}$, significant differences $(p<0.05)$ in bond strength were observed between the novel dithiooctanoate monomers (2-MEDT, 6-MHDT, and
10-MDDT) (19.0-20.5 MPa) and the conventional sulfurcontaining monomers (BMEDS, BMPDS, BMDDS, VBATDT, and MPMA) (0-6.8 MPa). On bonding to Ag, the dithiooctanoate monomers exhibited significantly higher bond strengths $(38.8-43.5 \mathrm{MPa})$ than the conventional sulfur-containing monomers (0 $\mathrm{MPa})$ $(p<0.05)$. On bonding to $\mathrm{Pt}$ and $\mathrm{Pd}$, significant differences in bond strength were observed between the dithiooctanoate monomers and BMEDS, VBATDT, and MPMA $(p<0.05)$.

On bonding to $\mathrm{Au}$ alloy, the bond strengths of 2-MEDT, 6-MHDT, and 10-MDDT were significantly higher than that of conventional sulfur-containing monomers of BMEDS, BMPDS, VBATDT, and MPMA $(p<0.05)$. On bonding to Ag alloy and Au-Ag-Pd alloy, the bond strengths of all the novel dithiooctanoate monomers were significantly higher than all the conventional sulfur-containing monomers $(p<0.05)$.

Table 2 Effects of dithiooctanoate monomers and conventional sulfur-containing monomers on tensile bond strength (TBS) to precious metals, using modified MMA-PMMA/BPO-DEPT resin, after 2,000 thermal cycles

\begin{tabular}{|c|c|c|c|c|}
\hline \multirow{2}{*}{$\begin{array}{l}\text { Sulfur-containing } \\
\text { monomer }\end{array}$} & \multicolumn{4}{|c|}{ TBS [mean (standard deviation)] to precious metals } \\
\hline & $\mathrm{Au}$ & $\mathrm{Ag}$ & $\mathrm{Pt}$ & $\mathrm{Pd}$ \\
\hline 2-MEDT & $19.2(4.1)^{\mathrm{a}}$ & $38.8(5.5)^{\mathrm{a}}$ & $33.1(3.2)^{\mathrm{a}}$ & $18.9(3.9)^{\mathrm{a}}$ \\
\hline 6-MHDT & $19.0(6.6)^{\mathrm{a}}$ & $40.1(6.2)^{\mathrm{a}}$ & $33.0(5.2)^{\mathrm{a}}$ & $30.8(4.0)^{\mathrm{a}}$ \\
\hline 10-MDDT & $20.5(5.6)^{\mathrm{a}}$ & $43.5(5.8)^{\mathrm{a}}$ & $33.6(4.2)^{\mathrm{a}}$ & $31.3(4.3)^{\mathrm{a}}$ \\
\hline BMEDS & 0 & 0 & $21.8(1.2)^{\mathrm{b}}$ & $11.6(3.3)^{\mathrm{b}}$ \\
\hline BMPDS & $6.3(0.5)^{b}$ & 0 & $34.7(3.8)^{\mathrm{a}}$ & $21.1(3.8)^{\mathrm{a}}$ \\
\hline BMDDS & $6.5(0.8)^{b}$ & 0 & $34.4(4.2)^{\mathrm{a}}$ & $21.3(3.1)^{\mathrm{a}}$ \\
\hline VBATDT & $6.8(2.1)^{\mathrm{b}}$ & 0 & $14.6(2.2)^{\mathrm{c}}$ & $9.8(3.2)^{\mathrm{b}}$ \\
\hline MPMA & $1.6(0.8)^{\mathrm{c}}$ & 0 & $0.5(0.2)^{\mathrm{d}}$ & $6.9(2.6)^{b}$ \\
\hline 4-META (Control) & 0 & 0 & 0 & $2.6(0.5)^{\mathrm{c}}$ \\
\hline
\end{tabular}

Note: $n=5$.

Adhesive resin used: Modified MMA-PMMA/BPO-DEPT resin.

One thermal cycle: $4^{\circ} \mathrm{C}$ water for $1 \mathrm{~min}$ and $60^{\circ} \mathrm{C}$ water for $1 \mathrm{~min}$.

Groups from the same column that are identified with the same superscript letter are not significantly different $(p>0.05)$.

Table 3 Effects of dithiooctanoate monomers and conventional sulfur-containing monomers on tensile bond strength (TBS) to dental precious metal alloys, using modified MMA-PMMA/BPO-DEPT resin, after 2,000 thermal cycles

\begin{tabular}{|c|c|c|c|}
\hline \multirow{2}{*}{$\begin{array}{l}\text { Sulfur-containing } \\
\text { monomer }\end{array}$} & \multicolumn{3}{|c|}{ TBS [mean (standard deviation)] to dental precious metal alloys } \\
\hline & Au alloy & Ag alloy & Au-Ag-Pd alloy \\
\hline 2-MEDT & $40.4(6.5)^{\mathrm{a}}$ & $38.8(3.5)^{\mathrm{a}}$ & $39.2(4.4)^{\mathrm{a}}$ \\
\hline 6-MHDT & $41.2(7.8)^{\mathrm{a}}$ & $30.0(4.7)^{\mathrm{a}}$ & $40.2(6.3)^{\mathrm{a}}$ \\
\hline 10-MDDT & $45.5(7.1)^{\mathrm{a}}$ & $31.2(4.6)^{\mathrm{a}}$ & $40.9(5.6)^{\mathrm{a}}$ \\
\hline BMEDS & $23.1(3.3)^{\mathrm{b}}$ & $10.4(2.1)^{\mathrm{b}}$ & $24.1(3.8)^{\mathrm{b}}$ \\
\hline BMPDS & $28.2(2.8)^{\mathrm{b}}$ & $13.7(3.1)^{\mathrm{b}}$ & $23.9(2.6)^{b}$ \\
\hline BMDDS & $36.1(3.3)^{\mathrm{a}}$ & $28.3(3.0)^{\mathrm{b}}$ & $32.5(3.5)^{\mathrm{b}}$ \\
\hline VBATDT & $25.8(4.2)^{\mathrm{b}}$ & $25.2(3.5)^{\mathrm{c}}$ & $27.5(3.2)^{b}$ \\
\hline MPMA & $3.7(0.3)^{\mathrm{c}}$ & $21.4(3.6)^{\mathrm{c}}$ & $11.3(2.4)^{\mathrm{c}}$ \\
\hline 4-META (Control) & 0 & $10.3(2.2)^{\mathrm{b}}$ & $15.5(2.5)^{\mathrm{c}}$ \\
\hline
\end{tabular}

Note: $n=5$.

Adhesive resin used: Modified MMA-PMMA/BPO-DEPT resin.

One thermal cycle: $4^{\circ} \mathrm{C}$ water for $1 \mathrm{~min}$ and $60^{\circ} \mathrm{C}$ water for $1 \mathrm{~min}$.

Groups from the same column that are identified with the same superscript letter are not significantly different $(p>0.05)$. 


\section{DISCUSSION}

The use of metal primers for the fabrication of seating cast restorations and fixed dental prostheses has increased substantially ${ }^{28)}$. To develop more advanced metal primers, this study was undertaken to evaluate the bonding performances of three novel dithiooctanoate monomers (namely, 2-MEDT, 6-MHDT, and 10-MDDT) ${ }^{26}$ ) on adhesion to seven kinds of precious metals and alloys and then compare their bond performance results against five sulfur-containing monomers. The conventional sulfur-containing monomers selected for comparison in this study were three non-cyclic disulfides (i.e., BMEDS, BMPDS, and BMDDS $)^{24)}$, a triazinedithione $\left(\right.$ VBATDT $^{25)}$, and a thiophenol (MPMA) ${ }^{23)}$. For a non sulfur-containing monomer, the acidic adhesive monomer of 4-META was selected as the control monomer. For luting, a modified MMA-PMMA/BPO-DEPT resin was used. As shown in Fig. 1, the chemical structures of all the sulfur-containing adhesive monomers typically comprised three parts: a polymerizable functional group, a connecting group, and an adhesion-promoting group containing sulfur atom(s) ${ }^{29)}$. Despite the commonality of possessing the typical three parts in their structures, significant differences $(p<0.05)$ in bond strength were observed for adhesion to $\mathrm{Au}, \mathrm{Ag}, \mathrm{Au}$ alloy, $\mathrm{Ag}$ alloy, and $\mathrm{Au}-\mathrm{Ag}-\mathrm{Pd}$ alloy between the three novel dithiooctanoate monomers and the five conventional sulfur-containing monomers.

On the significant differences $(p<0.05)$ in bond strength between the three cyclic disulfide compounds (dithiooctanoate monomers) and the non-cyclic disulfide compounds, it was thought that their bond strengths were attributed to the ability of chemical interaction derived from their structures. For the cyclic disulfide monomers, findings of previous surface-enhanced Raman scattering (SERS) spectroscopy studies ${ }^{19,20)}$ showed that organic sulfur compounds were chemically adsorbed onto $\mathrm{Au}$, leading to the formation of monolayer films by a spontaneous assembly of organic thiols on the Au surface. Leveraging on this finding ${ }^{19,20)}$, it was plausible to suggest that the cyclic disulfide group of 6,8-dithiooctanoate moiety underwent the cleavage of the disulfide bonds (-S-S-) intermolecularly on the Au surface. The reaction

\section{Cyclic disulfide}
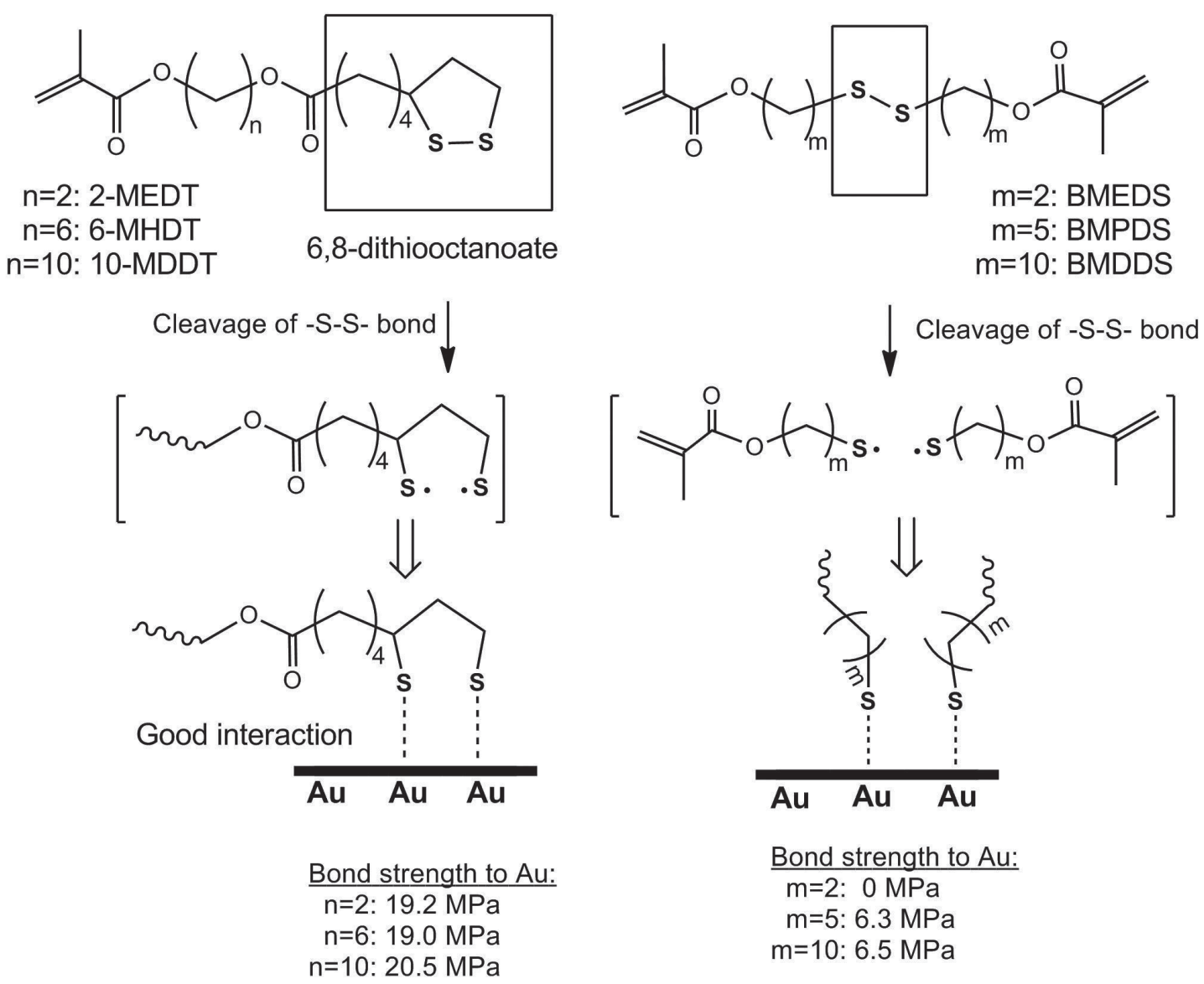

Fig. 2 Plausible chemical interaction of sulfur-containing adhesive monomers on the Au surface. Both dithiooctanoate monomers and non-cyclic disulfide monomers undergo cleavage of the -S-S- bond, and interacted chemically with Au atoms to form -S-Au on the Au surface. 
products were absorbed and interacted immediately with $\mathrm{Au}$ atom to form -S-Au bond on the metal surface (Fig. 2) ${ }^{28,29)}$. For the non-cyclic disulfide monomers, their bond strengths to precious metal and alloys decreased in the following order: BMDDS>BMPDS>BMEDS. They might have also undergone similar cleavage of the -S-Sbond, resulting in a chemical interaction with $\mathrm{Au}$ atoms to form -S-Au bond on the Au surface (Fig. 2). Although it remained to be elucidated the differences in chemical reaction between cyclic dithiooctanoate monomers and non-cyclic disulfide monomers with $\mathrm{Au}$, it was clearly shown in this study that the bond strength of dithiooctanoate monomers to Au was significantly higher than that of non-cyclic disulfide monomers $(p<0.05)$.

On the bond strengths of triazinedithione monomer (VBATDT) and thiophenol monomer (MPMA) to precious metals and alloys, they were found to be significantly lower $(p<0.05)$ than the three dithiooctanoate monomers. As per the above argument for non-cyclic disulfide monomers, it was thought that their chemical structures accounted for the host of differences in chemical interaction and polymerization reactivity between dithiooctanoate monomers and VBATDT or MPMA. In particular, the low bond strengths obtained by MPMA were caused by adverse polymerization reactivity with acrylic resin-based adhesives because the thiols acted as so-called chain transfer agents ${ }^{30)}$. For VBATDT, a contradicting result was obtained in a previous study ${ }^{30}$ ) which reported that VBATDT was effective for bonding to precious metals. In the case of VBATDT, the influence of its chemical structure on bond strength seemed to be rather ambivalent because of the presence of tautomeric structures such as triazinedithione and triazinedithiol $^{20,25)}$. In a study by Suzuki et al. ${ }^{20)}$ which investigated the tensile bond strength of MMA-PMMA/ TBBO resin to $\mathrm{Au}$, it was found that pretreatment with a metal primer which comprised 0.5 wt\% of VBATDT yielded 25.7 MPa after 2,000 times of thermal cycling. This value did not agree with the bond strength of VBATDT to $\mathrm{Au}(6.8 \mathrm{MPa})$ in this study, although modified MMA-PMMA/BPO-DEPT resin was used instead. These findings seemed to suggest that polymerization initiators had influence on adhesion. However, for the novel dithiooctanoate monomers of 2-MEDT, 6-MHDT, and 10-MDDT, their bond strengths with modified MMA-PMMA/BPO-DEPT resin in this study were comparable with those obtained by eight kinds of novel dithiooctanoate monomers with MMA-PMMA/TBBO resin in our previous study ${ }^{26)}$. The mean tensile bond strength of 10-MDDT to Au was 20.5 $\mathrm{MPa}$, as compared to that of $6.5 \mathrm{MPa}$ by a conventional sulfur-containing monomer BMDDS, and even $0 \mathrm{MPa}$ by 4-META. On the whole, the bond strength values of all tested monomers to precious metals and alloys seemed to be of such a tendency: 10-MDDT>BMDDS>4-META. This trend considerably correlated with the degree of chemical adsorption and reactivity of adhesive monomers on the $\mathrm{Au}$ surface. In other words, the bonding performance of an adhesive monomer was dependent on its degree of chemical interaction with $\mathrm{Au}$, which in turn was dependent on the chemical structure. In the context of this study, 4-META was an acidic compound which rendered it incapable of bonding to precious metals. Consequently, the poor chemical bonding of 4-META with $\mathrm{Au}$ atoms caused the bonded specimens to fail during thermal cycling.

In dental prosthetic practices, sandblasting is frequently used as a surface roughening treatment prior to the bonding of resin composites to alloys ${ }^{3)}$ or the bonding of metal alloys to enamel ${ }^{31)}$. This is because sandblasting increases the surface areas of metals and their mechanical retentive forces, hence increasing the bond strength to metals. However, for intraoral repair of fractured porcelain- or composite-veneers, sandblasting is not a convenient treatment method in the oral environment. In the present study, the mean tensile bond strength of 10-MDDT to Au alloy, without sandblasting treatment, was $45.5 \mathrm{MPa}$. Therefore, this study showed that 10-MDDT exhibited higher bonding performance than conventional sulfur-containing adhesive monomers even without any prior surface roughening treatment. To preclude the influence of mechanical retentive forces caused by surface irregularities, highly smooth metal surfaces (polished up to 4,000 grit) were used in this study. Apart from showing the difference in bonding durability between dithiooctanoate monomers and conventional sulfurcontaining monomers, results obtained in this study also showed that the bonding ability of adhesive monomers to metals could be unbiasedly evaluated using highly smooth metal surfaces after exposure to 2,000 times of thermal cycling.

The appearance of the three novel dithiooctanoate monomers was a yellowish liquid. When compared with conventional sulfur-containing monomers such as thiols, thiophenols, and thiophosphoric acids, the novel dithiooctanoate monomers possess inherent advantages such as absence of bad odor and good stability both in air and in organic solvents (such as acetone and ethanol). These properties are highly desirable improvements over the conventional sulfur-containing monomers for application to commercial products. Indeed, 10-MDDT, which was shown in this study to exhibit superior bonding performance, was already included in the formulation of a commercial primer, Metal Link Primer (M.L. Primer, Shofu Inc., Kyoto, Japan) in 2003. In a recent study by Okuya et al. ${ }^{32)}$, it was favorably reported that the highest shear bond strengths of M.L. Primer to $\mathrm{Au}$ and high-gold content alloy after 2,000 times of thermal cycling were 33.5 MPa and 33.3 MPa respectively.

Apart from the utilization of 10-MDDT in Metal Link Primer, the newly developed dithiooctanoate adhesive monomers have been patented as "(meth) acrylic ester derivatives" which can provide high and durable adhesion to precious metals and alloys ${ }^{26)}$. The next step forward is to explore the feasibility of combining the newly developed dithiooctanoate monomers with acidic adhesive monomers and examine the effect thereof on adhesion to precious metals, precious metal alloys, and non-precious metal alloys. 


\section{CONCLUSIONS}

Based on the findings in this study, the following conclusions were drawn.

1. On bonding to $\mathrm{Au}$ and $\mathrm{Ag}$, the novel dithiooctanoate monomers of 2-MEDT, 6-MHDT, and 10-MDDT exhibited significantly higher tensile bond strengths than the conventional sulfur-containing monomers (BMEDS, BMPDS, BMDDS, VBATDT, MPMA) and acidic adhesive monomer 4-META $(p<0.05)$.

2. On bonding to $\mathrm{Au}$ alloy, $\mathrm{Ag}$ alloy, and Au-Ag-Pd alloy, all the three novel dithiooctanoate monomers showed significantly higher tensile bond strengths than the conventional sulfur-containing monomers (except BMDDS) and 4-META $(p<0.05)$.

3 . When compared with the conventional sulfurcontaining monomers tested in this study, the novel dithiooctanoate monomers exhibited excellent bonding to both precious metals and alloys.

\section{REFERENCES}

1) Yoshida K, Matsumura H, Atsuta M. Monomer composition and bond strength of light-cured 4-META opaque resin. J Dent Res 1990; 69: 849-851.

2) Matsumura H, Shimoe S, Nagano K, Atsuta M. Effect of noble metal conditioners on bonding between prosthetic composite material and silver-palladium-copper-gold alloy. J Prosthet Dent 1999; 81: 710-714.

3) Antoniadou M, Kern M, Strub JR. Effect of a new metal primer on the bond strength between a resin cement and two high-noble alloys. J Prosthet Dent 2000; 84: 554-560.

4) Tanaka T, Nagata K, Takeyama M, Atsuta M, Nakabayashi N, Masuhara E. 4-META opaque resin - a new resin strongly adhesive to nickel-chromium alloy. J Dent Res 1981; 60: 1697-1706.

5) Tanaka T, Fujiyama E, Shimizu H, Takaki A, Atsuta M. Surface treatment of nonprecious alloys for adhesion-fixed partial dentures. J Prosthet Dent 1986; 55: 456-462.

6) Matsumura H, Nakabayashi N. Adhesive 4-META/ MMA-TBB opaque resin with poly(methyl methacrylate)coated titanium dioxide. J Dent Res 1988; 67: 29-32.

7) Matsumura H, Tanaka T, Taira Y, Atsuta M. Bonding of a cobalt-chromium alloy with acidic primers and tri- $n$ butylborane-initiated luting agents. J Prosthet Dent 1996; 76: 194-199.

8) Matsumura H, Tanaka T, Atsuta M. Bonding of silverpalladium-copper-gold alloy with thiol derivative primers and tri- $n$-butylborane initiated luting agents. J Oral Rehabil 1997; 24: 291-296.

9) Musil R, Tiller HJ. Molecular coupling of resin veneers to alloy surfaces. Dent Labor 1984; 32: 1155-1161.

10) Chang JC, Powers JM, Hart D. Bond strength of composite to alloy treated with bonding systems. J Prosthodont 1993; 2: 110-114.

11) Kern M, Thompson VP. Sandblasting and silica-coating of dental alloys: volume loss, morphology and changes in the surface composition. Dent Mater 1993; 9: 155-161.

12) Tanaka T, Hirano M, Kawahara H, Matsumura H, Atsuta M. A new ion-coating surface treatment of alloys for dental adhesive resins. J Dent Res 1988; 67: 1376-1380.

13) Kadoma Y. Adhesive properties and kinetic polymerization behavior of resins containing adhesion promoting monomers for precious metals. Dent Mater J 2005; 24: 335-341.

14) Eder A, Wickens J. Surface treatment of gold alloys for resin adhesion. Quintessence Int 1996; 27: 35-40.

15) Gates WD, Diaz-Arnold AM, Aquilino SA, Ryther JS Comparison of the adhesive strength of a BIS-GMA cement to tin-plated and non-tin-plated alloys. J Prosthet Dent 1993; 69: $12-16$.

16) Dixon DL, Breeding LC, Hughie ML, Brown JS. Comparison of shear bond strengths of two resin luting systems for a base and a high noble metal alloy bonded to enamel. J Prosthet Dent 1994; 72: 457-461.

17) Caeg C, Leinfelder KF, Lacefield WR, Bell W. Effectiveness of a method used in bonding resins to metal. J Prosthet Dent 1990; 64: 37-41.

18) Ohno H, Araki Y, Endo K. A new method for promoting adhesion between precious metal alloys and dental adhesives. J Dent Res 1992; 71: 1326-1331.

19) Suzuki M, Miyazaki T, Hisamitsu H, Kadoma Y, Morioka $Y$. Study on chemical reaction of methylthiirane on gold colloid by surface-enhanced Raman scattering. Langmuir 1999; 15: 7409-7410.

20) Suzuki M, Yamamoto M, Fujishima A, Miyazaki T, Hisamitsu H, Kojima K, Kadoma Y. Raman and IR studies on adsorption behavior of adhesive monomers in a metal primer for $\mathrm{Au}, \mathrm{Ag}$, $\mathrm{Cu}$, and Cr surfaces. J Biomed Mater Res 2002; 62: 37-45.

21) Colin DB, Troughton EB, Tao YT, Evall J, Whitesides GM, Nuzzo RG. Formation of monolayer films by the spontaneous assembly of organic thiols from solution onto gold. J Am Chem Soc 1989; 111: 321-335.

22) Nuzzo RG, Dubois LH, Allara DL. Fundamental studies of microscopic wetting on organic surfaces. 1. Formation and structural characterization of a self-consistent series of polyfunctional organic monolayers. J Am Chem Soc 1990; 112: 558-569.

23) Kojima K. Studies on adhesion of functional monomers with SH group to tooth substrates and dental alloys. J J Dent Mater 1986; 5: 92-105.

24) Kadoma Y, Kojima K. Adhesive properties of MMA-PMMA/ TBBO resin to dental alloys treated with sulfide-containing monomer. J J Dent Mater 1992; 11: 940-946.

25) Mizuno A, Toda Y, Itoh M, Kojima K, Kadoma Y. NMR analysis of thione-thiol tautomerization of triazine derivative in the solid state and in acetone solution. J Mol Structure 1998; 441: 149-153.

26) Kojima K, Kadoma Y, Ikemura K. (Meth)acrylic ester derivatives. United States Patent No. 5,670,657 (1997).

27) Ikemura K, Endo T. Syntheses of adhesive-promoting monomers bearing carboxylic moieties and their bonding performance with new polymerization initiators comprising 5-monosubstituted barbituric acids. J Adhes Soc Jpn 1998; 34: 138-149.

28) Koizumi H, Ishii T, Naito K, Yoneyama T, Tanoue N, Matsumura H. Effects of triazine dithione and hydrophobic phosphate monomers on bonding to $\mathrm{Ag}-\mathrm{Pd}-\mathrm{Cu}-\mathrm{Au}$ alloy and titanium with a methacrylic resin-based luting agent. J Adhes Dent 2010; 12: 215-222.

29) Kadoma Y. Chemical structures of adhesion promoting monomers for precious metals and their bond strengths to dental metals. Dent Mater J 2003; 22: 343-358.

30) Matsumura H, Kamada K, Tanoue N, Atsuta M. Effect of thione primers on bonding of noble metal alloys with an adhesive resin. J Dent 2000; 28: 287-293.

31) Sen D, Nayir E, Pamuk S. Comparison of the tensile bond strength of high-noble, noble, and base metal alloys bonded to enamel. J Prosthet Dent 2000; 84: 561-566.

32) Okuya N, Minami H, Kurashige H, Murahara S, Suzuki S, Tanaka T. Effect of metal primers on bonding of adhesive resin cement to noble alloys for porcelain fusing. Dent Mater J 2010; 29: 177-187. 